\title{
TERC polymorphisms are associated both with susceptibility to colorectal cancer and with longer telomeres
}

\author{
A M Jones, ${ }^{1}$ A D Beggs, ${ }^{1}$ L Carvajal-Carmona, ${ }^{1}$ S Farrington, ${ }^{2}$ A Tenesa, ${ }^{2}$ M Walker, ${ }^{2}$ \\ K Howarth, ${ }^{1}$ S Ballereau, ${ }^{2}$ S V Hodgson, ${ }^{3}$ A Zauber, ${ }^{4}$ M Bertagnolli, ${ }^{5}$ R Midgley, ${ }^{6}$ \\ H Campbell, ${ }^{7}$ D Kerr, ${ }^{6}$ M G Dunlop, ${ }^{2}$ I P M Tomlinson ${ }^{1}$
}

\begin{abstract}
- Additional materials are published online only. To view these files please visit the journal online (http://gut.bmj. com/content/61/2.toc).

${ }^{1}$ Wellcome Trust Centre for Human Genetics, University of Oxford, Oxford, UK

${ }^{2}$ Colon Cancer Genetics Group Institute of Genetics and

Molecular Medicine, University of Edinburgh and MRC Human Genetics Unit, Edinburgh, UK ${ }^{3}$ Department of Clinical Genetics, St George's Hospital Medical School, London, UK ${ }^{4}$ Department of Epidemiology and Biostatistics, Memorial Sloan-Kettering Cancer Center New York, New York, USA ${ }^{5}$ Brigham and Women's Hospital, Dana Farber Cancer Institute, Boston,

Massachusetts, USA

${ }^{6}$ Department of Clinical

Pharmacology, University of

Oxford, Oxford, UK

${ }^{7}$ Public Health Sciences,

University of Edinburgh,

Edinburgh, UK
\end{abstract}

\section{Correspondence to}

Professor lan Tomlinson, Wellcome Trust Centre for Human Genetics, University of Oxford, Roosevelt Drive, Oxford OX3 7BN, UK;

iant@well.ox.ac.uk

Revised 4 May 2011 Accepted 29 May 2011 Published Online First 27 June 2011

This paper is freely available online under the BMJ Journals unlocked scheme, see http:// gut.bmj.com/site/about/ unlocked.xhtml

\section{ABSTRACT}

Background and aims Shorter telomeres have been associated with increased risk of malignancy, including colorectal cancer (CRC). Telomere length is heritable and may be an intermediate phenotype linked to genetic susceptibility to CRC.

Methods In a large sample, the study investigated whether candidate single nucleotide polymorphisms (SNP) in 'telomere biology' genes were associated with telomere length in leucocytes. SNP associated with an increased risk of CRC were searched for separately. Results Carriers of the common allele at SNP rs 10936599, near the telomerase RNA component (TERC) locus, had significantly longer telomeres. It was independently found that the same rs10936599 allele was associated with increased risk of both CRC and colorectal adenomas. Neither telomere length nor CRC risk was associated with variation near telomerase reverse transcriptase or other telomere biology genes. In silico analysis showed that SNP rs2293607 was strongly correlated with rs10936599, mapped within TERC transcripts, had a predicted effect on messenger RNA folding and lay at a reported transcription factor binding site. TERC mRNA were expressed, differing only at the alleles of rs2293607, in CRC cell line HCT116. The longtelomere/CRC-risk allele was associated with higher levels of TERC mRNA and the formation of longer telomeres.

Conclusions Common genetic variation at TERC is associated with both longer telomeres and an increased risk of CRC, a potential mechanism being reduced levels of cell senescence or death. This finding is somewhat paradoxical, given retrospective studies reporting that CRC cases have shorter telomeres than controls. One possibility is that that association actually results from poorer survival in patients with longer telomeres.

Chromosome telomeres consist of multiple short repeats (TTTAGG) that protect against large-scale genetic rearrangements. In most somatic cells, telomeres shorten with cell divisions, eventually leading to cellular senescence. Telomere length decreases as humans age, ${ }^{1}$ and exceptionally longlived individuals have relatively long telomeres. ${ }^{2}$ Some patients have inherited tendencies to short telomeres, resulting in diseases that include dyskeratosis congenita, ${ }^{3}$ idiopathic pulmonary fibrosis ${ }^{4}$ and aplastic anaemia. ${ }^{5} 6$

In some human cells, including stem cells, telomere length is maintained by the expression of the

\section{Significance of this study}

What is already known about this subject?

- In retrospective, studies, shorter telomeres have been found to be markers of, or causative for, colorectal cancer (CRC) risk.

- We have found a number of common inherited polymorphisms to be associated with CRC risk.

- Polymorphisms near the telomerase reverse transcriptase (TERC) gene have been associated with telomere length.

What are the new findings?

- The major allele at polymorphism rs 10936599 is associated with an increased risk of colorectal carcinomas and adenomas, but is also associated with longer telomeres.

- rs10936599 is strongly correlated with another polymorphism, rs2293607, within the TERC gene.

- TERC mRNA that differ only by the alleles at rs2293607 have different expression levels and the major allele causes longer telomeres in vitro, as predicted by the genetic association data.

- There therefore exists a paradox in that TERC polymorphisms have confirmed associations with both CRC and longer telomeres, yet shorter telomeres have classically been associated with cancer risk.

How might it impact on clinical practice in the foreseeable future?

- Constitutionally longer telomeres may predispose to CRC.

- The relationship between shorter telomere length and CRC risk is in doubt.

- It is possible that the reported relationship may result from effects on prognosis rather than susceptibility.

- CRC risk prediction using genetic data may be more reliable under some circumstances than reliance on intermediate biomarkers.

telomerase enzyme. ${ }^{7} 8$ The holoenzyme's core activity is provided by telomerase reverse transcriptase (TERT), which utilises the telomerase RNA component (TERC) as a template to add repeats to the existing telomeres. A hallmark of 
cancer is a continuing ability to proliferate and/or avoid death. Somatic activation of telomerase helps to confer these features in many tumour types. In cervical cancer, for example, it has been proposed that telomerase expression relies on high levels of $T E R C$ that result from the amplification of chromosome 3q26.2. ${ }^{9}$

Telomere length is a heritable trait, ${ }^{10-12}$ and a small number of polymorphisms have been associated with telomere length. ${ }^{13} 14$ The hypothesis that shorter telomeres predispose to cancer in the general population initially gained support from an increased risk of cancer ${ }^{15-18}$ in some of the diseases associated with very short telomeres (see above), presumably as a consequence of 'chromosomal instability'. Several studies have since reported that leucocyte telomeres are shorter in cancer cases than in controls, ${ }^{1}{ }^{19-24}$ although the data are inconsistent among studies and tumour types. Previous studies on colorectal cancer (CRC) have produced conflicting results regarding associations between leucocyte telomere length and CRC risk. Prospective studies ${ }^{24-27}$ have found no convincing association between telomere length and CRC risk (see supplementary table 1 , available online only). In contrast, the retrospective arm of the study of Pooley et $a^{26}$ found a strong association between shorter telomeres and CRC risk ( $\mathrm{N}=2249$ cases and 2161 controls, OR quartile1v4 $2.14,95 \%$ CI 1.77 to 2.59 ). Together with the same authors' prospective data, this finding raises the possibility that the retrospective association reflected not disease risk, but effects of the cancer itself and/or anticancer therapies and/or effects of telomere length on survival.

In order to acquire sufficient samples to empower analysis, studies have examined telomere length in leucocytes and no study has examined telomeres in the colorectum with regard to CRC risk. O'Sullivan et $a l^{1}$ and Risques et al ${ }^{28}$ measured telomeres in normal colonocytes in a relatively small sample set; they found not only the expected telomere shortening with age, but also some evidence of longer telomeres in the oldest individuals in the study. However, other studies on normal colonic mucosa from CRC patients have failed to find this biphasic relationship. ${ }^{29} 30$ O'Sullivan et $a l^{1}$ and Risques et $a l^{28}$ did not relate colonocyte telomere length to that in leucocytes.

We have searched for associations between variants in telomere pathway genes and telomere length in leucocytes. We have related the findings to data from the same samples, obtained as part of a genome-wide association study of CRC predisposition. ${ }^{31}$ The results demonstrate the existence of an association between telomere length and CRC predisposition, but show that this association is more complex than previously suspected.

\section{METHODS \\ Study participants}

Three sets of samples were taken from the general population or from individuals without a cancer history ${ }^{31}$ : (1) 2489 population controls (mean age 44 years, $49 \%$ men) from the Wellcome Trust Case-Control Consortium 2 National Blood Service (NBS) cohort (http://www.wtccc.org/; (2) 968 subjects (mean age 51 years, $52 \%$ men) from the Scottish population for the COGS study; and (3) 455 UK CORGI study controls (mean age 58 years, $41 \%$ men), these being individuals with a spouse or partner with CRC, but themselves unaffected by cancer and without a family history (to second degree relative level) of colorectal neoplasia.

CRC patients were from three sources: ${ }^{31}$ (1) 717 cases (mean age 67 years, 64\% men) from the VICTOR study, a phase III, randomised controlled trial of rofecoxib after potentially curative therapy; (2) 888 early-onset (age $\leq 55$ years) Scottish cases (mean age 49 years, 51\% men) from COGS; and (3) 552 familial CRC cases (mean age 63 years, 48\% men) from CORGI. For VICTOR, $51 \%$ of CRC were stage II, all the remainder being stage III; $62 \%$ of VICTOR cases received 5-fluorouracil-based chemotherapy and $8.3 \%$ had radiotherapy. Blood samples were taken a median of 127 weeks after surgery (interquartile range 100-170). For COGS, 19\% of cancers were stage I, 31\% stage II, $32 \%$ stage III and $18 \%$ stage IV; $28 \%$ of COGS patients received chemotherapy and $8 \%$ radiotherapy, and blood was sampled a median of 20 weeks after diagnosis (interquartile range 12-33). As CORGI samples were collected from cancer genetics clinics, they were highly variable in terms of their sampling dates post-diagnosis; data on cancer stage and chemotherapy were similarly variable in terms of their availability.

For studies of genetic susceptibility to CRC only, we additionally analysed 496 cases (mean age 62 years, 59\% men) from the QUASAR2 study of adjuvant capecitabine with or without bevacizumab in stage II/III CRC. We excluded QUASAR2 samples from the telomere length studies, because blood was generally taken from these patients during the period of chemotherapy, potentially leading to considerable confounding effects from neutropaenia and other relative or absolute changes in the leucocyte count.

A total of 1569 individuals of white European ancestry with multiple (two to 10 ) or 'significant' $(>1 \mathrm{~cm}$ diameter or villous histology) colorectal adenomas was identified from the Adenoma Prevention with Celecoxib Trial.

All DNA was extracted from whole peripheral blood. Single nucleotide polymorphism (SNP) genotyping, quality control and imputation were performed according to standard procedures outlined in the supplementary material, available online only. All studies were performed in accordance with local research ethics committee approval (Southampton and South-West Hampshire REC (A) 06/O1702/99).

\section{Telomere assays and quality control}

Telomere length was measured using minor modifications of Cawthon's monochrome multiplex quantitative PCR method (details in Cawthon), ${ }^{32}$ which we found to provide far greater consistency than other previously published methods; 98 samples were assayed in duplicate and for 93 of these, the telomere length measurement lay within $15 \%$ of each other and only two lay outside $25 \%$. All samples were analysed by study of origin in 96-well plate format together with five control dilutions of a single, anonymous standard DNA, which was used to fit linear regression lines for the $\log$ (telomere) and $\log (\mathrm{HBC}$ control) Ct values and DNA concentration for each plate. Very good fits were observed (see supplementary figure 1, available online only). For each sample on that plate, the $\mathrm{Ct}$ value for the control probe was used to determine the amount of DNA in the reaction from the standard line. From this value, the expected $\mathrm{Ct}$ for the telomere was determined. The expected telomere $\mathrm{Ct}$ value was then subtracted from that observed for that sample according to the standard line, to give a relative telomere measurement (CorrDCt) for each sample on each plate. In summary, CorrDCt was the measured telomere length for each sample relative to that of the anonymous standard sample; the larger CorrDCt, the greater was the measured telomere length.

\section{Assessment of functional effects of TERC variant}

HCT116 CRC cells, a near-diploid line homozygous for the A allele at rs2293607, were used in this experiment. TERC messenger RNA was PCR-amplified using primers TCCCTTTATAAGCCGACTC and ATTCATTTTGGCCGACTT, 
so as to encompass TERC transcripts AI825849 and AW207347. This was cloned into pcDNA3.1 and clones of identical sequence, apart from the A and G alleles at rs2293607, were obtained by site-directed mutagenesis. HCT116 cells were transfected with this construct using Lipofectamine 2000 under standard conditions. Cells were co-transfected with pEGFP-C3 so as to measure transfection efficiency using GFP expression. This confirmed high $(>90 \%)$ efficiency in all cases. Cells transfected with empty pcDNA3.1 and pEGFPc3 were used as controls. Based on pilot experiments that estimated the time of maximum TERC expression, we measured telomere lengths after $48 \mathrm{~h}$. At the same time, we measured total TERC expression using Taqman realtime quantitative assay Hs03297287_s1 (Applied Biosystems), with SF3B1 expression as a control. All experiments were performed in triplicate.

\section{RESULTS \\ Search for polymorphisms associated with telomere length in leucocytes: confirmation of SNP near TERC but not TERT}

Using a candidate locus approach, we identified from public databases all SNP within $50 \mathrm{~kb}$ of a panel of genes proposed by Mirabello et $a l^{33}$ as 'telomere biology' candidates (see supplementary table 2, available online only). One hundred and twenty-eight tagging SNP in these regions (see supplementary table 3, available online only) had been genotyped in our cases and controls in all samples. We used real-time quantitative PCR to determine the normalised telomere length in cases and controls from each of the following sample sets (CORGI, COGS, NBS, VICTOR). Data were stratified by case/control status, sample set, age and sex. Using a threshold for association of $\mathrm{p}=10^{-4}$, approximating to a false discovery rate of 0.01 , we found five SNP (rs16854453, rs10936599, rs11709840, rs1920116, rs10936603) to be associated with telomere length (table 1). These SNP were all within a region of approximately $90 \mathrm{~kb}$ on chromosome 3q26.2 (170,974,795-171,062,665 bases), were in strong or moderate pairwise linkage disequilibrium (LD) (see supplementary figure 2, available online only), and lay within a haplotype block that encompassed the TERC locus (chr3: 170965092-170965542). We found that they were in strong LD with a SNP (rs12696304) proximal to TERC that had previously been reported to be associated with telomere length. ${ }^{13}$

No other 'telomere biology' SNP was associated with telomere length, the strongest association $\left(p=2.0 \times 10^{-3}\right)$ being at a SNP, rs27061, distal to TERT. The failure to find an association between TERT SNP and telomere length confirms the data of other groups ${ }^{13} 34$ and is of note, because SNP at this site (rs401681, rs4635969, rs2736100, rs4975616, rs2736098) have previously been associated with cancer risk ${ }^{35}$; each of these five SNP or a proxy $\left(r^{2} \sim 1.0\right)$ was typed in our samples.

\section{TERC SNP are associated with increased risk of CRC}

At the same time as our study of telomere length in CRC patients, we were undertaking a genome-wide association study of CRC. The latest stage of the genome-wide association study based on 11 case-control cohorts, ${ }^{31}$ including several that sampled incident cases, found that rs10936599 showed a significant association with CRC risk $\left(p=2.55 \times 10^{-8}, O R_{\text {per allele }} 0.93\right.$, 95\% CI 0.91 to 0.96 ), independent of age and sex. Table 2 shows rs10936599 genotype counts in the sample sets used in this study. As rs10936599 is a synonymous SNP within an exon of myoneurin (MYNN), we had reported MYNN as the gene close to rs10936599 most likely to harbour functional variation. ${ }^{31}$ However, our finding that rs10936599 was associated with telomere length prompted a more detailed investigation. We initially confirmed that, although the other four TERC SNP associated with telomere length were not genotyped in all 11 case-control cohorts, each showed evidence of association with CRC risk (see supplementary table 4, available online only). As the major allele at rs10936599 was associated with both longer telomeres and CRC risk, the genetic analysis strongly suggested that, if leucocyte telomeres are a valid proxy for those in colonocytes, longer telomeres were associated with an increased risk of CRC.

\section{rs10936599 Genotype is associated with risk of colorectal adenomas}

Telomerase activity has generally been associated with carcinomas rather than their benign precursors. However, we wondered whether the effect of rs10936599 might also be mediated in normal tissue or in early tumorigenesis (eg, by effects in normal stem cells). We therefore examined whether rs10936599 alleles were associated with disease in patients with colorectal adenomas who had not developed CRC. Cases were derived from the Adenoma Prevention with Celecoxib adenoma prevention trial; ${ }^{36}$ controls were from the publicly available 1958 UK Birth Cohort. ${ }^{37}$ After stratifying for a family history of CRC, the major allele at rs 10936599 was associated with an increased risk of adenomas (table 2; OR $0.883,95 \%$ CI 0.80 to 0.98 ; $\left.\chi_{1}^{2}=5.30, \mathrm{p}=0.011\right)$. The variation close to TERC thus probably acts, at least partly, at an early stage in tumorigenesis.

\section{Evidence that the variation tagged by rs10936599 acts through telomere length}

Although we thought it highly plausible that rs10936599 alleles affected CRC risk through telomere length, it was possible that the functional variation associated with rs10936599 acted through another mechanism and that the association with telomere length was an epiphenomenon. Given the potential confounding effect on telomere length of patients having CRC or receiving treatment for their disease ${ }^{26}$ and in the absence of prospective cohort data, we could not test this alternative hypothesis by directly examining rs10936599 genotypes and telomere lengths in our CRC cases and controls. We therefore undertook an indirect test. Assuming that telomere length in leucocytes is a proxy for that in colonocytes, we reasoned the following: if the influence of rs10936599 genotype on CRC risk

Table 1 SNP associated with telomere length

\begin{tabular}{lllllllll}
\hline SNP & Chr & Position & N & p Value & p (R) & Beta & Beta (R) & $\mathbf{0}$ \\
\hline rs16854453 & 3 & 171041936 & 4 & $3.38 \times 10^{-6}$ & $4.86 \times 10^{-5}$ & -0.067 & -0.066 & 0.321 \\
rs10936599 & 3 & 170974803 & 5 & $3.92 \times 10^{-5}$ & $3.92 \times 10^{-5}$ & -0.055 & -0.055 & 0.580 \\
rs11709840 & 3 & 171052943 & 4 & $4.14 \times 10^{-5}$ & $4.14 \times 10^{-5}$ & -0.059 & -0.059 & 0.528 \\
rs1920116 & 3 & 171062673 & 5 & $4.19 \times 10^{-5}$ & $4.19 \times 10^{-5}$ & -0.055 & -0.055 & 0.0 \\
rs10936603 & 3 & 171028354 & 5 & $4.53 \times 10^{-5}$ & $7.39 \times 10^{-4}$ & -0.055 & -0.053 & 0.0 \\
\hline
\end{tabular}

Beta, In(OR); Chr, chromosome; $I^{2}$, test of heterogeneity; Position, single nucleotide polymorphism (SNP) location in base pairs in NCBI genome Build 36; $p$ (R), fixed effects $p$ value in linear regression meta-analysis; $p$ Value, fixed effects $p$ value in linear regression meta-analysis; 0 ,Cochran's 0 statistic. 
Table 2 Risk of CRC and colorectal adenoma associated with the rs10936599 SNP

\begin{tabular}{llllllllll}
\hline Series & Ca11 & Ca12 & Ca22 & Co11 & Co12 & Co22 & MAF ca & MAF co & OR \\
\hline V058 & 69 & 413 & 731 & 150 & 932 & 1407 & 0.23 & 0.25 & 0.894 \\
UK1/CORGI & 30 & 162 & 360 & 25 & 162 & 268 & 0.20 & 0.23 \\
Scotland1/COGS & 50 & 301 & 537 & 69 & 383 & 516 & 0.23 & 0.27 \\
APC adenomas/NBS & 75 & 558 & 936 & 152 & 936 & 1412 & 0.23 & 0.23 \\
\hline
\end{tabular}

Genotype counts in cases (Ca) and controls (Co) are shown by sample series.

Allele $1=\mathrm{A}$, Allele $2=\mathrm{G}$

Meta-analysis of the three colorectal cancer (CRC) case-control series gives $0 R 0.85(95 \% \mathrm{Cl} 0.78$ to 0.93$), p=1.57 \times 10^{-4}, p_{\text {het }}=0.442$.

Note that only the data from the sample sets in this study are shown; the full genome-wide association study data can be obtained from Jeanclos et al. ${ }^{31}$

APC, Adenoma Prevention with Celecoxib Trial; MAF, minor allele frequency; NBS, National Blood Service; SNP, single nucleotide polymorphism.

is not mediated through telomere length, the effect size of rs10936599 should not vary with telomere length; any variation in the effect size of rs10936599 with telomere length would be most likely to result from a direct effect of longer telomeres on CRC risk, for example, by pushing those with generally long telomeres over some threshold at which cancer risk was particularly increased. In order to standardise telomere lengths among the case sample sets (CORGI, VICTOR, COGS), we ranked each individual according to the length of their telomeres in that sample set. We then used logistic regression analysis to determine the effect size of rs10936599 according to the rank of the individuals in each case data set compared with all controls, stratifying by sex, age and study of origin. We initially observed that when cases with relatively short telomeres (ie, in the first and/or second quartiles) were studied, the effect of the rs10936599 genotype was not significant, whereas a significant effect was seen in the third quartile and a highly significant effect $\left(\ln (\mathrm{OR})-0.43, p=5.04 \times 10^{-8}\right)$ was seen in the fourth quartile. More generally, we tested whether rs10936599 effect size was related to ranked telomere length by regressing $\ln (\mathrm{OR})$ on telomere decile; a significant linear relationship was found (In $(\mathrm{OR})(-0.0418 \times$ decile $)+0.0561 ; \mathrm{R}^{2}=0.5882 ; \mathrm{t}=-3.38, \mathrm{p}=0.010$; see supplementary figure 3 , available online only). While the underlying origin of this association is unclear, its existence suggests that rs10936599 directly affects CRC risk through telomere length.

\section{Annotation and fine mapping of the $3 q 26.2$ region}

From HapMap two data (http://www.hapmap.org/), we found that rs10936599 (chr3: 170,974,795) is in strong or moderate LD with SNP within the region chr3: 170,941,263-171,012,592 that includes the genes TERC, ARPM1, MYNN and LRRC34. Using 1000genomes project data (http://www.1000genomes.org/), 44 SNP within this region were found to be correlated with rs10936599 with $\mathrm{r}^{2}>0.5$ (see supplementary table 5, available online only).

The only SNP fulfilling these LD criteria that was both within or close to TERC and had promising functional annotation was rs2293607 (chr3: 170,965,029; $\mathrm{r}^{2}=1.0$ with rs10936599, http:// www.biomart.org/). The most abundant TERC transcript maps to chr3: $170,965,092-170,965,542$, but two other TERC transcripts contain rs2293607 (AI825849, chr3: 170,965,004170,965,446 and AW207347, chr3: 170,965,002-170,965,373). rs2293607 was predicted by RNAfold (http://rna.tbi.univie.ac.at/ cgi-bin/RNAfold.cgi) to alter the transcript's secondary mRNA structure (see supplementary figure 4, available online only). Moreover, data from the ENCODE project (http://genome.ucsc. edu/cgi-in/hgTrackUi ?hgsid $=174313571 \& \mathrm{c}=$ chr15\&g=wgEncodeReg) indicated two histone marks (H3K4Me1 and H3K4Me3) in the immediate vicinity of rs2293607. The same region was also DNaseI-hypersensitive and the predicted binding site of multiple transcription factors, including NFKB, PU.1, POU2F2 and MYC
(http://genome.ucsc.edu/cgi-bin/hgTrackUi?hgsid=174313571\& $\mathrm{c}=$ chr15\&g $=$ wgEncodeReg).

In order to refine the location of the causal variation in the region around TERC, we inspected association signals between SNP genotype and telomere length and CRC susceptibility, following imputation of SNP using the 1000genomes project as a reference. In general, the association plots for the two variables mirrored each other (see supplementary figure 5, available online only), and consequently the effect sizes were very strongly correlated $(t=27.6, p<0.001)$. In both cases, the strongest signal was found at approximately $171.05 \mathrm{Mb}$, although the associations at that site were only marginally stronger than those present in a larger region that included rs2293607 between approximately $170.90 \mathrm{Mb}$ and $171.05 \mathrm{Mb}$.

\section{In-vitro analysis of the effects of rs2293607 genotypes in TERC transcripts}

As a result of the possible effects of rs2293607 on TERC RNA structure or transcription, we performed functional assays on TERC transcripts that varied only at the A (major, CRC risk, longer telomere) and G (minor, CRC protection, shorter telomere) alleles at this site. In order to examine effects in cells of large bowel origin, we used the CRC cell line HCT116 as a model system. We expressed exogenous TERC transcripts that varied only at the $\mathrm{A} / \mathrm{G}$ polymorphic site, performing each experiment in triplicate and controlling for potential differences in transfection efficiency between experiments. Initially, we quantified the expression of total (endogenous and exogenous) TERC mRNA for each of the two variants in comparison with empty vector and untransfected controls. We found that after $48 \mathrm{~h}$, total TERC expression was higher in cells expressing the exogenous TERC, and that cells with the A allele construct expressed higher TERC than those with the G allele (figure 1).

We then examined whether the increased TERC expression associated with the A allele had functional consequences. Although no gross differences in HCT116 morphology or behaviour were observed at the $48 \mathrm{~h}$ time point, we did not necessarily expect these in a transformed cell line. We therefore assayed telomere length in cells with the A and G allele transcripts. This showed that, in concordance with our hypothesis, expression of the A allele TERC transcript resulted in longer telomeres in HCT116, whereas the G allele had little effect on telomeres compared with the control cells (figure 1).

\section{Telomere length in CRC cases versus controls}

Given the prediction from our work above that CRC risk is associated with longer telomeres, yet pre-existing evidence suggests that CRC cases have shorter telomeres than controls, ${ }^{26}$ we compared telomere length in our three sets of CRC cases (VICTOR, CORGI and COGS) and in the three sets of controls (CORGI, COGS and NBS). Telomere length (CorrDCt) was approximately normally distributed (see supplementary figure 6, 

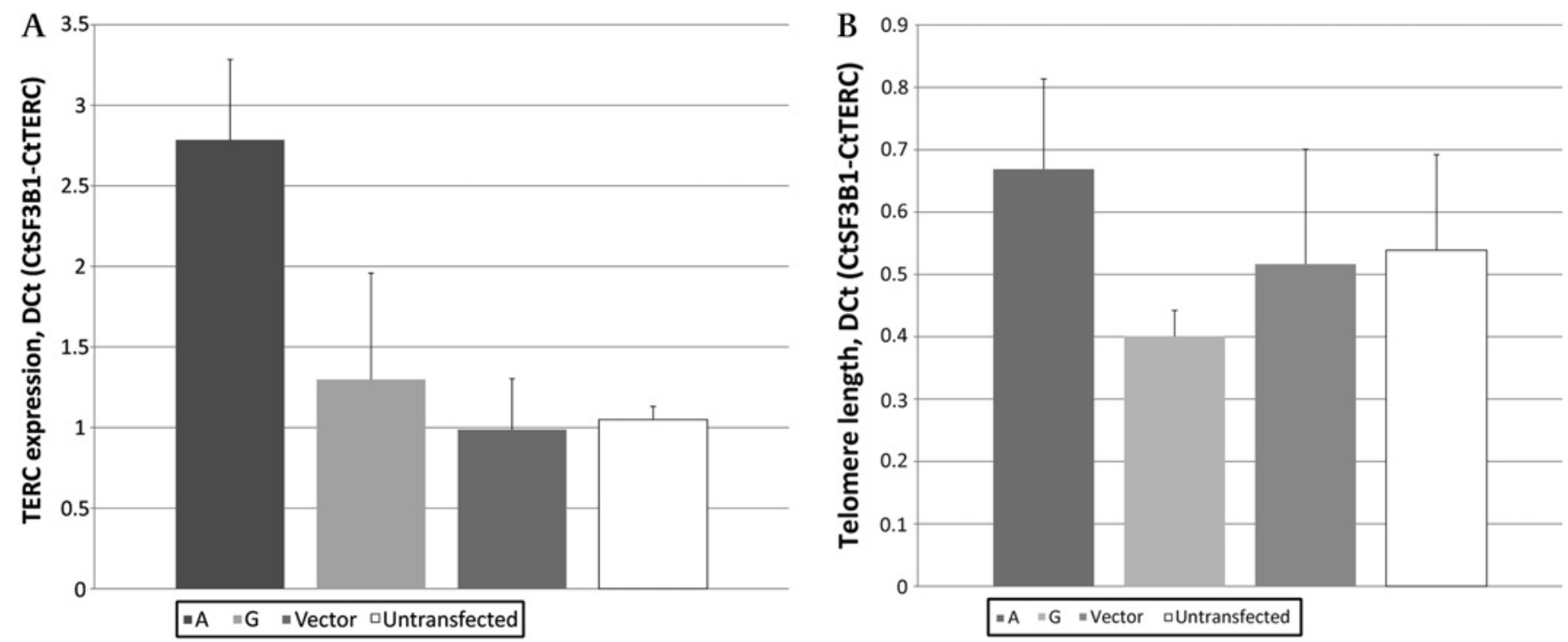

Figure 1 Telomerase RNA component expression (A) and telomere length (B) in HCT116 after transfection with telomerase RNA component constructs carrying the $A$ and $G$ alleles at rs2293607, compared with empty vector and untransfected cells. Error bars show SEM of experiments performed in triplicate.

available online only) and decreased with age (see supplementary figure 7 , available online only). Incorporating age, sex and study of origin as covariates, we performed logistic regression analysis to compare case and control telomere lengths. We used NBS controls as a comparison with VICTOR because of the absence of unaffected controls from the clinical trial sample set. Contrary to expectations from our genetic data, but consistent with previous retrospective studies, we found that cases had significantly shorter telomeres than controls (mean CorrDct ${ }_{\text {cases }}$ 1.04, mean CorrDCt $t_{\text {controls }}$ 1.11), although there was also considerable variation between sample series (figure 2). Overall, after stratifying for age, sex, sample set and rs10936599 genotype, telomeres were significantly shorter in cases than controls (OR $0.71,95 \%$ CI 0.56 to $0.90, z=2.85, p=4.37 \times 10^{-3}$ ).

The CORGI and VICTOR study cases were collected months, or occasionally years, after diagnosis and treatment. We therefore compared telomere lengths in these two sets of cases with those in the COGS cases, which were collected closer to diag-

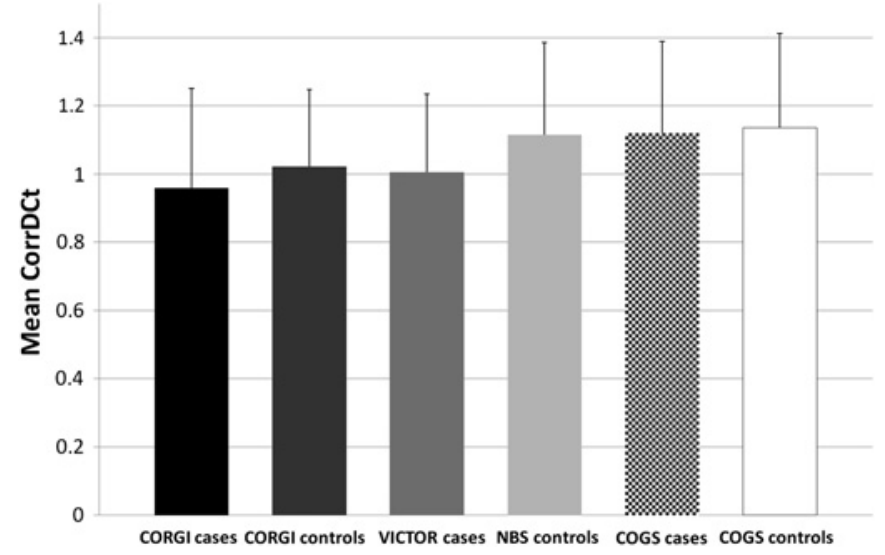

Figure 2 Telomere lengths in cases and controls from each sample set. The bar chart shows mean CorrDCt for each sample set (error bars show standard deviations). Note the shorter telomeres in cases than controls, with the exception of the COGS cases, which have similar lengths to the some other control sample sets, although shorter lengths than their corresponding controls. nosis. After controlling for age, sex and rs10936599 genotype, the COGS samples had significantly longer telomeres than the other samples $\left(p=4.22 \times 10^{-6}\right.$, logistic regression analysis). We then explored possible causes for this finding. We used multiple linear regression to search within VICTOR - as this was a highquality clinical trial dataset with full clinical and follow-up data-for associations between telomere length and the following: (1) use of chemotherapy (all 5-fluorouracil-based) or rofecoxib; (2) use of radiotherapy; (3) blood sampling time after surgery and (4) CRC stage. After controlling for age and sex, we found no association between telomere length and chemotherapy $(p=0.88)$, rofecoxib $(p=0.87)$, radiotherapy $(p=0.053)$, sampling time $(p=0.18)$ or stage $(p=0.42)$. There was, moreover, no association between recurrence-free survival and telomere length (HR 0.94, $\mathrm{p}=0.87$, Cox proportional hazards model) after correcting for stage, radiotherapy, chemotherapy, age and sex (details not shown).

\section{DISCUSSION}

We have confirmed that SNP close to the TERC locus are associated with leucocyte telomere length in humans, using methods that were different from those in the previous studies of Codd et $a l^{13}$ and Levy et al. ${ }^{14}$ We also found independently that the same TERC SNP are associated with susceptibility to CRC. However, the SNP data show that, contrary to expectations, individuals with longer telomeres are predicted to be at increased risk of CRC.

The causal variation close to TERC and its mode of action remain to be identified, although it is most likely to lie within the region $170.90 \mathrm{Mb}-171.05 \mathrm{Mb}$. We investigated the candidate SNP rs2293607 as a result of its genetic association with rs10936599 and rs12696304, together with its predicted effects on mRNA structure and/or expression. Our evidence suggests that rs2293607 might indeed have functional effects on TERC expression and thus on telomere length. If increased CRC risk does directly result from long telomeres, one plausible model is that telomere lengthening increases a normal cell's stem-like properties and thus its chances of acquiring tumour-causing mutations, consistent with the increased risk of both adenomas and carcinomas associated with rs10936599. 
Despite the genetic evidence predicting that longer telomeres increase CRC risk, evidence from retrospective studies, such as our own, has shown that telomeres in peripheral blood cells are shorter in CRC cases than controls. However, associations between telomere length and CRC risk have not been shown in prospective studies, despite such studies probably including individuals with occult cancers. Although the prospective studies may have been too small to detect associations between telomere length and cancer reliably, the data as a whole suggest that the association between short telomeres and CRC in retrospective studies is in some way the result of disease or differential survival rather than the cause. Our samples taken closer to diagnosis (COGS study) had longer telomeres than those taken months or years after diagnosis (CORGI or VICTOR). These data are consistent with an association between longer telomeres and poorer survival, or with telomere attrition in the aftermath of treatment. However, our three sets of CRC cases differed in several ways other than sampling time, such as the therapeutic regimens used, inclusion criteria and ethnicity. We therefore also searched for evidence that telomere length depended on time between surgery and sampling or recurrence-free survival in VICTOR, because it was a clinical trial with excellent follow-up data. However, we found no association in either case. We also found no evidence that 5-fluorouracil-based chemotherapy or radiotherapy was associated with shorter telomeres in VICTOR. Given the long time between surgery and sampling in VICTOR and consequent sampling bias against early recurrence, we suggest that a requirement is an analysis of telomere length and survival based on a set of CRC cases sampled close to diagnosis, ideally before chemotherapy, and with excellent follow-up data.

The rival models linking telomere length and colorectal tumorigenesis are shown in figure 3 . However, several further issues remain regarding telomeres, TERC and CRC risk.
1. Telomerase may repair DNA other than at telomeres, ${ }^{38-40}$ because de novo synthesis of telomere repeats may occur at double-stranded breaks. Despite our evidence to the contrary, it may be these extra-telomeric functions that are causally associated with increased CRC risk.

2. Telomere length is most conveniently measured in peripheral blood: this can lead to non-genetic variation between samples (eg, if the composition of the leucocyte count varies) and it may not reflect the situation in the target tissue for cancer development. In particular, we believe it most unlikely that circulating DNA shed from cancer cells that have short telomeres contributed anything other than a tiny proportion of the total blood DNA in which we measured telomere length in CRC cases. We attempted to minimise sampling issues by collecting in a similar fashion from cases and controls when possible (notably in CORGI and COGS). However, this had to be balanced against the practicalities of empowering the study with enough samples (eg, we used NBS controls as a comparison with VICTOR as a result of the absence of unaffected controls from those clinical trial sample sets). Data were analysed throughout having stratified by sample set.

3. There was overlap between the NBS samples analysed both in this study and that of Codd et al. ${ }^{13}$ However, even excluding these samples, the association between rs10936599 and telomere length remained (OR $0.673,95 \% \mathrm{CI} 0.511$ to $\left.0.886, p=5 \times 10^{-3}\right)$. In fact, the consistency between our work and that of Codd et al ${ }^{13}$ provides good evidence of the technical validity of our assays. ${ }^{32}$

4. It is possible that the association between telomere length and cancer risk is non-montonic, allowing shorter telomeres to be associated with CRC overall, yet the minor TERC allele to be protective.

5. It is a further conundrum that TERT tagSNP have been reported to be associated with an increased risks of lung,
Figure 3 Models of associations between telomere length and colorectal cancer (CRC) risk. Arrows indicate causality. (1) The current model is based on observation of shorter leucocytes telomeres in CRC cases than controls, assumes that this is mirrored in colorectal crypt stem cells and posits a causal link between shorter telomeres and CRC development, possibly through chromosomal instability. (2) Our model proposes that genetic factors cause longer telomeres, assumes that this is mirrored in colorectal crypt stem cells and proposes that longer telomeres directly influences CRC risk, possibly through effects on stem cell numbers or longevity. The shorter telomeres in retrospectively collected CRC arise as a result of disease or as a sample collection artefact caused by shorter survival in individuals with longer telomeres. (3) A third possibility must be borne in mind, namely that genetic factors cause longer telomeres, but that the genetic factors do not influence risk through telomere length, which is an epiphenomenon with no relevance or even a partially compensatory restraining influence on carcinogenesis; shorter telomeres of other origins may still cause an increased CRC risk. 
breast and other cancers, yet common genetic variants at TERT are not associated with telomere length in the studies undertaken to date. The reason underlying the organ or site-specific associations of cancers with TERC or TERT polymorphisms remains unclear.

Telomere length is a trait that is highly likely to be under stabilising selection. If telomeres are too short, they predispose to chromosomal aberrations and the diseases that result. However, they may also lead to earlier cell death, especially if DNA damage checkpoints are active. Very long-lived humans tend to have long telomeres, so what is the downside of having very long telomeres? Our work suggests that at least part of this downside is increased CRC risk, and there is evidence to support this contention in that chronically increased TERT expression promotes tumorigenesis in mouse models. ${ }^{41}$ Although some of the data are paradoxical, there is a clear link between rs10936599 and longer telomeres in leucocytes and increased risk of CRC. This finding may overcome some of the problems inherent in directly measuring telomere length in retrospectively collected cancer cases and controls. We propose that the previously reported associations between cancer risk and short telomeres in the general population wrongly emphasised the chromosomal instability aspects of cancer risk over the aspects relating to cell lifespan and stem-like properties.

Funding The Oxford group acknowledges funding from the EU FP7 CHIBCHA project, the Oxford Comprehensive Biomedical Research Centre and Cancer Research UK. The authors are also very grateful to Willem Ouwehand and colleagues for supplying the National Blood Service controls. The Edinburgh group were supported by grants from: Cancer Research UK (C348/A12076), Scottish Government Chief Scientist Office (K/OPR/2/2/D333), Medical Research Council (G0000657-53203) and a Centre Grant from CORE as part of the Digestive Cancer Campaign. Core infrastructure support to the Wellcome Trust Centre for Human Genetics, Oxford was provided by grant 090532/Z/09/Z

Correction notice This article has been modified since it was published online first. The funding statement was corrected.

Competing interests None

Ethics approval This study was conducted with the approval of the Southampton and South-West Hampshire REC (A).

Contributors Molecular analysis: AMJ, KH, MW, SF; data analysis: AMJ, ADB, LCC, IPMT; study design and sample collection: HC, AZ, MB, DK, SVH, MGD, IPMT.

Provenance and peer review Not commissioned; externally peer reviewed.

\section{REFERENCES}

1. O'Sullivan J, Risques RA, Mandelson MT, et al. Telomere length in the colon declines with age: a relation to colorectal cancer? Cancer Epidemiol Biomarkers Prev 2006:15:573-7

2. Atzmon G, Cho M, Cawthon RM, et al. Evolution in health and medicine Sackler colloquium: genetic variation in human telomerase is associated with telomere length in Ashkenazi centenarians. Proc Natl Acad Sci U S A 2010;107(Suppl 1):1710-17.

3. Walne AJ, Vulliamy T, Beswick R, et al. TINF2 mutations result in very short telomeres: analysis of a large cohort of patients with dyskeratosis congenita and related bone marrow failure syndromes. Blood 2008;112:3594-600.

4. Cronkhite JT, Xing C, Raghu G, et al. Telomere shortening in familial and sporadic pulmonary fibrosis. Am J Respir Crit Care Med 2008;178:729-37.

5. Du HY, Pumbo E, Ivanovich J, et al. TERC and TERT gene mutations in patients with bone marrow failure and the significance of telomere length measurements. Blood 2009;113:309-16.

6. Vulliamy T, Beswick R, Kirwan M, et al. Mutations in the telomerase component NHP2 cause the premature ageing syndrome dyskeratosis congenita. Proc Natl Acad Sci U S A 2008;105:8073-8.

7. Blasco MA. Telomere length, stem cells and aging. Nat Chem Biol 2007;3:640-9.

8. Flores I, Canela A, Vera $\mathrm{E}$, et al. The longest telomeres: a general signature of adult stem cell compartments. Genes Dev 2008;22:654-67.

9. Soder Al, Hoare SF, Muir S, et al. Amplification, increased dosage and in situ expression of the telomerase RNA gene in human cancer. Oncogene 1997; 14:1013-21.
10. Bischoff C, Graakjaer J, Petersen $\mathrm{HC}$, et al. The heritability of telomere length among the elderly and oldest-old. Twin Res Hum Genet 2005;8:433-9.

11. Jeanclos $\mathbf{E}$, Schork NJ, Kyvik KO, et al. Telomere length inversely correlates with pulse pressure and is highly familial. Hypertension 2000;36:195-200.

12. Slagboom PE, Droog S, Boomsma DI. Genetic determination of telomere size in humans: a twin study of three age groups. Am J Hum Genet 1994;55:876-82.

13. Codd V, Mangino $M$, van der Harst $P$, et al. Common variants near TERC are associated with mean telomere length. Nat Genet 2010;42:197-9.

14. Levy D, Neuhausen SL, Hunt SC, et al. Genome-wide association identifies OBFC1 as a locus involved in human leukocyte telomere biology. Proc Natl Acad Sci U S A 2010;107:9293-8.

15. Perona R, Machado-Pinilla R, Manguan C, et al. Telomerase deficiency and cancer susceptibility syndromes. Clin Trans/ Oncol 2009;11:711-14.

16. Calado RT, Regal JA, Hills $M$, et al. Constitutional hypomorphic telomerase mutations in patients with acute myeloid leukemia. Proc Natl Acad Sci U S A 2009;106:1187-92.

17. Alter BP, Giri N, Savage SA, et al. Cancer in dyskeratosis congenita. Blood 2009;113:6549-57.

18. Scheinberg $\mathbf{P}$, Cooper JN, Sloand EM, et al. Association of telomere length of peripheral blood leukocytes with hematopoietic relapse, malignant transformation, and survival in severe aplastic anemia. JAMA 2010;304:1358-64.

19. Barwell J, Pangon L, Georgiou A, et al. Is telomere length in peripheral blood lymphocytes correlated with cancer susceptibility or radiosensitivity? $\mathrm{Br} \mathrm{J}$ Cancer 2007;97:1696-700.

20. Broberg K, Bjork J, Paulsson K, et al. Constitutional short telomeres are strong genetic susceptibility markers for bladder cancer. Carcinogenesis 2005;26:1263-71.

21. Prescott J, McGrath $\mathrm{M}$, Lee IM, et al. Telomere length and genetic analyses in population-based studies of endometrial cancer risk. Cancer 2010;116:4275-82.

22. Rosenberg R, Gertler $R$, Stricker $D$, et al. Telomere length and hTERT expression in patients with colorectal carcinoma. Recent Results Cancer Res 2003:162:177-81.

23. Yoon SL, Jung SI, Do EJ, et al. Short rare hTERT-VNTR2-2nd alleles are associated with prostate cancer susceptibility and influence gene expression. BMC Cancer 2010;10:393

24. Zee RY, Castonguay AJ, Barton NS, et al. Mean telomere length and risk of incident colorectal carcinoma: a prospective, nested case-control approach. Cancer Epidemiol Biomarkers Prev 2009:18:2280-2.

25. Lee IM, Lin J, Castonguay AJ, et al. Mean leukocyte telomere length and risk of incident colorectal carcinoma in women: a prospective, nested case-control study. Clin Chem Lab Med 2010:48:259-62.

26. Pooley KA, Sandhu MS, Tyrer J, et al. Telomere length in prospective and retrospective cancer case-control studies. Cancer Res 2010:70:3170-6.

27. Willeit $\mathbf{P}$, Willeit J, Mayr $\mathrm{A}$, et al. Telomere length and risk of incident cancer and cancer mortality. JAMA 2010;304:69-75.

28. Risques RA, Lai LA, Brentnall TA, et al. Ulcerative colitis is a disease of accelerated colon aging: evidence from telomere attrition and DNA damage. Gastroenterology 2008; 135:410-18.

29. Nakamura K, Furugori E, Esaki $Y$, et al. Correlation of telomere lengths in normal and cancers tissue in the large bowel. Cancer Lett 2000;158:179-84.

30. Rampazzo E, Bertorelle R, Serra L, et al. Relationship between telomere shortening genetic instability, and site of tumour origin in colorectal cancers. $\mathrm{Br} J$ Cancer 2010; 102:1300-5.

31. Houlston RS, Cheadle J, Dobbins SE, et al. Meta-analysis of three genome-wide association studies identifies susceptibility loci for colorectal cancer at 1q41, 3q26.2 $12 q 13.13$ and 20q13.33. Nat Genet 2010;42:973-7.

32. Cawthon RM. Telomere length measurement by a novel monochrome multiplex quantitative PCR method. Nucleic Acids Res 2009;37:e21.

33. Mirabello L, Yu K, Kraft P, et al. The association of telomere length and genetic variation in telomere biology genes. Hum Mutat 2010;31:1050-8.

34. Pooley KA, Tyrer J, Shah M, et al. No association between TERT-CLPTM1L single nucleotide polymorphism rs401681 and mean telomere length or cancer risk. Cance Epidemiol Biomarkers Prev 2010;19:1862-5.

35. Baird DM. Variation at the TERT locus and predisposition for cancer. Expert Rev Mol Med 2010;12:e16.

36. Bertagnolli MM, Eagle CJ, Zauber AG, et al. Celecoxib for the prevention of sporadic colorectal adenomas. N Engl J Med 2006;355:873-84.

37. Power C. A review of child health in the 1958 birth cohort: National Child Development Study. Paediatr Perinat Epidemiol 1992;6:81-110.

38. Hao ZM, Luo JY, Cheng J, et al. Intensive inhibition of hTERT expression by a ribozyme induces rapid apoptosis of cancer cells through a telomere lengthindependent pathway. Cancer Biol Ther 2005;4:1098-103.

39. Parkinson EK, Fitchett C, Cereser B. Dissecting the non-canonical functions of telomerase. Cytogenet Genome Res 2008;122:273-80.

40. Rahman R, Latonen L, Wiman KG. hTERT antagonizes p53-induced apoptosis independently of telomerase activity. Oncogene 2005;24:1320-7.

41. Artandi SE, Alson S, Tietze MK, et al. Constitutive telomerase expression promotes mammary carcinomas in aging mice. Proc Natl Acad Sci U S A 2002;99:8191-6. 